\title{
Leitos de saúde mental em hospitais gerais: o caso do Rio de Janeiro
}

\author{
Mental health beds in general hospitals: the case of Rio de Janeiro
}

Rodrigo Cunha Echebarrena', Paulo Roberto Fagundes da Silva²

DOI: 10.1590/0103-11042020E319

\begin{abstract}
RESUMO O objetivo deste artigo foi analisar o modelo de provisão de leitos de saúde mental em três hospitais gerais da cidade do Rio de Janeiro e sua relação com a Rede de Atenção Psicossocial. Foram realizadas entrevistas com gestores locais, um gestor municipal e dois ex-coordenadores nacionais de saúde mental. Os resultados apontaram que os hospitais estudados oferecem espaços restritivos, com predomínio de terapia farmacológica e forte influência do modelo biomédico. A presença dos leitos de saúde mental nos hospitais tem-se mostrado uma experiência bem-sucedida na melhoria do cuidado integral aos usuários. A interação com a Rede de Atenção Psicossocial foi apresentada como modelo de assistência preconizado, mas ainda enfrentando fragilidades em sua implementação. Os achados da pesquisa evidenciaram que a baixa implantação de leitos de saúde mental em hospital geral na cidade se deve a lacunas na formação de profissionais e ao estigma que faz com que gestores resistam em receber usuários com transtornos mentais em suas instituições. Modificações recentes na política nacional de saúde mental, no sentido de interromper o fechamento de leitos em hospitais psiquiátricos, devem acarretar alterações na direção da reforma psiquiátrica no País.
\end{abstract}

PALAVRAS-CHAVE Saúde mental. Hospitais gerais. Desinstitucionalização. Brasil.

\begin{abstract}
The aim of this article is to analyze the model of provision of mental health beds in three general hospitals in the city of Rio de Janeiro and its relationship with the psychosocial care network. Interviews were conducted with local managers, a municipal manager and two former national mental health coordinators. The results showed that the studied hospitals offer restrictive spaces, with a predominance of pharmacological therapy and a strong influence of the biomedical model. The presence of mental health beds in hospitals has proved to be a successful experience in improving comprehensive care for users. The interaction with the psychosocial care network was presented as a recommended care model, but still facing weaknesses in its implementation. The research findings showed that the low implementation of mental health beds in a general hospital in the city is due to gaps in the training of professionals and the stigma that makes managers resist receiving users with mental disorders in their institutions. Recent changes in the national mental health policy in the sense of interrupting the closure of beds in psychiatric hospitals should lead to changes in the direction of psychiatric reform in the country.
\end{abstract}

1 Secretaria Municipal de Saúde (SMS) - Rio de Janeiro (RJ), Brasil.

2 Fundação Oswaldo Cruz (Fiocruz), Escola Nacional de Saúde Pública Sergio Arouca (Ensp), Departamento de Ciências Sociais (DCS) - Rio de Janeiro (RJ), Brasil. pr.fagundes@terra.com.br
KEYWORDS Mental health. General hospitals. Deinstitutionalization. Brazil. 


\section{Introdução}

A desinstitucionalização é um dos principais marcos no atendimento de pessoas com transtornos mentais e em uso prejudicial de substâncias desde a segunda metade do século XX. É interpretada como uma política projetada para prevenir a incapacidade crônica, defender os direitos humanos e reduzir o custo do cuidado. Na atualidade, $\mathrm{o}$ atendimento comunitário é considerado mais humano e de maior qualidade em comparação aos cuidados baseados em instituições ${ }^{1}$. O Brasil tem implementado por mais de três décadas uma atenção em saúde mental inspirada nessa premissa, construindo uma Rede de Atenção Psicossocial (Raps) - Centros de Atenção Psicossocial (Caps), integração com a atenção primária, residências terapêuticas etc. -, que acarretou uma significativa redução do número de leitos em hospitais psiquiátricos ${ }^{2}$. Para a intervenção hospitalar em saúde mental, essa política implica a adoção de internações curtas em leitos psiquiátricos localizados em hospitais gerais para o caso de pacientes em situação de crise, as quais não possam ser manejadas no âmbito da rede comunitária extra-hospitalar.

Essa escolha é decorrente da constatação que a integração dos dispositivos de saúde mental na rede de saúde geral é um dos pontos de partida para qualquer processo de reforma. Quando as hospitalizações ocorrem dentro da rede de saúde geral, evitam-se os mecanismos de exclusão próprios das instituições psiquiátricas tradicionais ${ }^{3}$. Na Itália, por exemplo, o cuidado em saúde mental é ofertado em uma rede que inclui pequenas unidades psiquiátricas dentro de hospitais gerais, dispositivos residenciais e centros de saúde mental para pacientes externos que servem como polo ( $h u b$ ) de um sistema de base comunitária ${ }^{4}$.

A utilização de leitos psiquiátricos em hospitais gerais tem um efeito adicional de reduzir o estigma relacionado com os transtornos mentais, aumentar a transparência da prática em saúde mental e aumentar a atenção sobre a saúde física dos usuários 5 . Os dispositivos comunitários de atendimento devem ter relação coordenada com os hospitais gerais que fazem a internação psiquiátrica. Segundo Desviat e Moreno ${ }^{6}$, essa coordenação é fundamental para a congruência e atuação sinérgica de dispositivos e profissionais, assim como para garantir a continuidade do cuidado da comunidade ao hospital e vice-versa.

Apesar de a diretriz de substituir a internação em hospital psiquiátrico pela hospitalização em leitos de saúde mental em hospitais gerais fazer parte da Política Nacional de Saúde Mental emanada pelo Ministério da Saúde ${ }^{7}$, o número de leitos disponíveis para internação psiquiátrica em hospitais gerais no Brasil ainda é muito inferior aos leitos oferecidos em hospitais psiquiátricos (menos de $10 \%$ do total) ${ }^{8}$.

O propósito deste artigo é descrever como esse processo se dá na cidade do Rio de Janeiro, segunda cidade mais populosa do País e que tem um padrão similar ao quadro nacional em sua política de saúde mental com significativa redução de leitos psiquiátricos, sem a necessária ampliação dos Caps III e a conversão dos leitos psiquiátricos remanescentes para os hospitais gerais 9 . Para consecução desse objetivo, serão analisados o modelo de provisão de leitos de saúde mental em três hospitais gerais e sua relação com a Raps.

\section{Metodologia}

Trata-se de um estudo exploratório no formato de estudo de caso, que buscou analisar as condições de implantação e gestão de três enfermarias de saúde mental localizadas em hospitais gerais municipais do Rio de Janeiro. Foram realizadas entrevistas semiestruturadas face a face com três gestores locais das unidades hospitalares (identificados como Gestor Local A, B e C), complementadas com três entrevistas abertas com o gestor de nível central de saúde mental do município do Rio de Janeiro (Gestor Municipal) e com dois 
ex-coordenadores nacionais de saúde mental do Ministério da Saúde (Ex-coordenador Nacional A e B), identificados como informantes-chave em face do papel relevante desempenhado, respectivamente, no âmbito municipal e federal. Complementarmente, foram realizadas pesquisas documentais em documentos oficiais, portarias e bancos de dados do Sistema Único de Saúde (SUS) de acesso público.

A análise e a interpretação dos dados do material das entrevistas foram inspiradas no modelo proposto por Creswell10, que envolve organizar e preparar os dados para análise, obter um sentido geral das informações, organizar materiais em grupos, usar o processo de codificação para gerar uma descrição do cenário, prever como a descrição e os temas serão representados na narrativa qualitativa e, como passo final na análise de dados, fazer uma interpretação do significado dos dados. Foi utilizado o método de usar passagens narrativas para transmitir os resultados da análise, e realizada uma comparação de resultados com informações extraídas da literatura de forma a verificar se os resultados confirmam informações passadas ou divergem delas.

O projeto de pesquisa foi submetido aos Comitês de Ética em Pesquisa da Escola Nacional de Saúde Pública Sergio Arouca (CEP - Ensp) e da Secretaria Municipal de Saúde do Rio de Janeiro (CEP - SMSRJ). Foi aprovado sob o número 67276617.7.0000.5240 (CEP-ENSP) e 67276617.7.3001.5279 (CEP - SMSRJ).

\section{Resultados e discussão}

Em 2018, na cidade do Rio de Janeiro, existiam 811 leitos disponíveis para internação psiquiátrica. Destes, 379 estavam ocupados com pacientes de longa permanência institucional em hospitais psiquiátricos (mais de um ano de internação). Dos demais leitos, 378 estavam localizados em hospitais psiquiátricos; e 54 , em enfermarias de saúde mental em hospitais gerais. Esses últimos leitos encontravam-se localizados no Hospital Municipal Ronaldo Gazolla, no Hospital Municipal Evandro Freire, no Hospital Municipal Pedro II e no Hospital Municipal Lourenço Jorge. Essa última unidade foi excluída do estudo por não estar credenciada pelo Ministério da Saúde na ocasião da pesquisa.

O Hospital Municipal Ronaldo Gazolla possuía 15 leitos, sem distribuição prévia por gênero. Havia um leito para isolamento dentro da enfermaria que podia ser utilizado para manejo de situações especiais. Externamente, havia um saguão para recepção de visitantes e movimentação da equipe. A enfermaria, que ficava no térreo, contava com uma sala de TV e atividades, posto de enfermagem, consultórios e uma sala de coordenação de equipe.

O Hospital Municipal Evandro Freire contava com 15 leitos em sua enfermaria, que estava localizada no segundo andar do hospital, em um espaço com três quartos, um hall, uma pequena sala de equipe, um posto de enfermagem e uma sala de descanso para a equipe. A divisão por gênero acontecia de acordo com a demanda, respeitando a separação por quartos.

A enfermaria de saúde mental do Hospital Municipal Pedro II funcionava no oitavo andar do edifício com 19 leitos. Os quartos se dividiam por gênero, sendo dois quartos para homens e dois quartos para mulheres. Além desse espaço, a enfermaria contava com posto de enfermagem, um hall usado como ambiente de convivência e um refeitório que também é utilizado para atividades coletivas.

O processo de análise das entrevistas resultou na proposição de três categorias de análise que serão apresentadas a seguir:

1. O modelo organizacional e a interação com a Rede de Atenção Psicossocial;

2. O processo de cuidado;

3. A desinstitucionalização da atenção. 


\section{O modelo organizacional e a interação com a Rede de Atenção Psicossocial}

As internações em saúde mental na cidade são reguladas por meio do Sistema de Regulação de vagas (Sisreg) desenvolvido pelo Ministério da Saúde que permite gerenciar as vagas para internações hospitalares em toda a cidade. O Sisreg é responsável por otimizar as vagas existentes para internações psiquiátricas no município, monitorando o tempo de espera até a internação e priorizando os casos mais graves. Se for definida a internação, a central de regulação do município decide pela unidade de internação, podendo ser um leito em hospital geral ou psiquiátrico.

Segundo o gestor local:

[...] havendo leito disponível, a regulação faz esse controle, internando aqui, preferencialmente, pacientes em crise psiquiátrica e com comorbidade clínica, mas não necessariamente. No caso, quem faz isso é a regulação. Nós só disponibilizamos o leito e esperamos. (Gestor Local A).

A visão do entrevistado de que a ocupação dos leitos dos hospitais gerais ocorre, preferencialmente, com pessoas portadoras de patologias clínicas e psiquiátricas não foi corroborada pelos outros dois gestores locais que disseram que suas enfermarias funcionam como qualquer outra enfermaria psiquiátrica, não privilegiando casos com necessidades clínicas conjuntas às psiquiátricas. Segundo eles, o critério de internação é a presença de quadro psiquiátrico agudo, independentemente de manifestações clínicas:

A questão toda está baseada só no quadro mental para internação. Quando existe uma comorbidade, a maioria das vezes os pacientes estão em outras clínicas do hospital e a equipe de saúde mental faz uma avaliação nas enfermarias. (Gestor Local B).

Como aqui é um hospital geral, muitas vezes nós recebemos um paciente que tenha comorbidade clínica. Mas não necessariamente tem que apresentar um quadro clínico concomitante para estar aqui na saúde mental. (Gestor Local C).

Em 2012, a área técnica de saúde mental do Ministério da Saúde mostrava interesse em delimitar o campo de intervenção dos leitos de saúde mental e emitiu nota técnica explicitando que os leitos se conformavam como pontos de atenção para garantir o acesso dos usuários à tecnologia hospitalar, particularmente no manejo do cuidado às intercorrências clínicas. Ao mesmo tempo, reafirmava que o Caps III era o ponto de atenção estratégico no cuidado e responsabilização pelas situações de crise ${ }^{11}$.

Uma dificuldade apontada pelos gestores locais foi a necessidade de internar a clientela de diversos pontos da cidade e de outros municípios.

Nós não nos restringimos a área programática. A demanda é de toda a cidade e até de outros municípios porque não existem serviços que comportem a população de pacientes dessas regiões. (Gestor Local B).

Segundo o gestor municipal,

A orientação que o regulador da central de internações tem é internar perto de onde o usuário mora. Mas a distribuição dos recursos na cidade é muito desigual, então você não pode vincular somente $a$ isso, você precisa olhar a rede como um todo. Se o paciente é de outro município e tem indicação de internação, será internado, mas com posterior retorno ao município de origem. (Gestor Municipal).

A Portaria GM n ${ }^{\circ}$ 148/ 2012 define que a assistência nas enfermarias de saúde mental deve acontecer de forma integrada à Raps, visando à continuidade da assistência e à corresponsabilidade pelos casos ${ }^{7}$. A distância entre os territórios de origem dos pacientes e as enfermarias foi apontada como problema para o desenvolvimento de um protocolo de cuidado mais intensivo. 
As equipes dos hospitais gerais com leitos de saúde mental adotam como práxis a comunicação da internação às equipes do território do paciente.

Normalmente assim que o paciente chega nós identificamos o território de procedência para a situação da rede local: Caps ou saúde da família e já começamos a pensar uma articulação para o projeto terapêutico, enquanto ele ainda está internado. (Gestor Local B).

As ações adotadas a partir daí variam muito em função das características dos territórios e da distância entre estes e a enfermaria. Um dos entrevistados, falando sobre a relação com os Caps, fala:

Nem todos os Caps vem; só os que estão mais próximos. [...] O projeto de saída é feito, muitas vezes em conjunto, outras não porque a equipe não consegue vir discutir o caso. (Gestor Local B).

Essa constatação pode denotar um modelo de atenção à crise pautado na baixa interlocução entre as redes de saúde mental e urgência, com um insuficiente protagonismo dos dispositivos comunitários no manejo das crises tal como é apontado por Dias e associados ${ }^{\mathbf{1 2}}$. Esses autores diferenciam as concepções da área de urgência e emergência da abordagem psicossocial: a primeira seria pautada na formalização técnica, especialmente na forma de protocolos; a segunda priorizaria a singularidade de intervenções a partir da avaliação de cada caso.

Mesmo com as dificuldades apontadas, as equipes procuravam que as internações fossem breves, e as altas eram dadas com o referenciamento do paciente a um local de tratamento próximo a sua moradia. Assim como redes extra-hospitalares capazes de atender de forma efetiva pacientes em quadros agudos podem reduzir de maneira significativa os encaminhamentos feitos para serviços de internação integral, as internações mais curtas são adequadas quando se dispõe de uma rede de recursos comunitários bem organizadas para recepção dos pacientes após a alta hospitalar ${ }^{13}$. Algumas dificuldades no momento da alta foram relatadas. Os pacientes dos três hospitais recebiam alta e levavam apenas as prescrições das medicações, com medicação para um dia de tratamento. A continuidade do tratamento medicamentoso depende da rápida absorção do paciente por algum equipamento da rede de saúde.

Os Caps destacam-se como dispositivo central de assistência a casos graves, mas os serviços de atenção básica vêm dividindo a responsabilidade do cuidado de forma significativa. A cidade do Rio de Janeiro teve importante crescimento de sua atenção básica. Em 2008, sua cobertura era de 3,5\% da população. Em 2013, a cobertura de atenção básica no município passou para $40 \%$. Soranz e colaboradores ${ }^{14}$ apontam que houve uma recaracterização da antiga Rede de Atenção à Saúde de atenção primária em um novo modelo centrado no Saúde da Família, que ampliou o escopo dos serviços oferecidos à população nesse nível de atenção. A ampliação da cobertura beneficiou também a atenção em saúde mental como pontuado por Soalheiro e associados ${ }^{\mathbf{1 5}}$ : os gestores das unidades de atenção básica mostraram-se sensíveis à inclusão das abordagens ao sofrimento mental pelas equipes de saúde da família, enfatizando a responsabilização pelo território.

Essa articulação territorial é fundamental para o desenvolvimento do cuidado além da internação. Como pontuam os ex-coordenadores nacionais de saúde mental:

Hospital geral é um ponto de assistência transitório. Você tem que ter uma rede de Caps potente. Não adianta o leito no hospital geral sem uma rede de Caps. Senão reproduz plenamente o hospital psiquiátrico. (Ex-coordenador Nacional A).

[...] a articulação com a rede secundária Caps e com a rede de atenção primária é vital para o funcionamento da unidade hospital geral. (Excoordenador Nacional B). 
Essas assertivas são corroboradas pelos resultados de um estudo realizado no período de 2008 a 2015 nas regiões metropolitanas dos municípios do Rio de Janeiro e de São Paulo, em que foram analisadas as internações psiquiátricas no SUS. Os resultados encontrados constataram a associação entre as tendências crescentes de oferta de Caps e atenção básica e a diminuição das taxas de internação nos grupamentos diagnósticos em avaliação nas regiões e período estudados ${ }^{16}$.

$\mathrm{O}$ enfrentamento às crises mais graves, passíveis de resultarem em internações hospitalares, passa pela organização da rede de Caps. Segundo o gestor municipal, seria desejável que, cada vez mais, a rede de atendimento à crise passasse pelo gerenciamento dos Caps e só casos mais complexos chegassem aos hospitais. Segundo o desenho adotado pelo município do Rio de Janeiro, o atendimento às crises deve acontecer territorialmente, com a participação da rede de Caps. Esse objetivo em 2016 carecia de maior resolutividade pela reduzida existência de Caps da tipologia III na composição da rede ( $24 \%$ do total).

$\mathrm{Na}$ visão de um ex-coordenador nacional de saúde mental:

[...] o serviço do hospital geral não substitui, nos mesmos termos, o hospital psiquiátrico. Ele se integra a uma rede, que essa sim substitui o modelo da tríade hospital psiquiátrico, internação e ambulatório. Era assim que funcionava o modelo manicomial, emergência, hospital, ambulatório. Era um tripé. A entrada do hospital geral na rede não substitui, mecanicamente, o hospital psiquiátrico. Ele se insere em uma rede dinâmica, que esta sim substitui o modelo hospitalocêntrico. (Excoordenador Nacional B).

Os leitos de saúde mental em hospital geral são contratualizados entre o município do Rio de Janeiro e as Organizações Sociais (OS) executantes dos serviços. Nesse modelo de gestão compartilhada, o município outorga o gerenciamento, a operacionalização e a execução das ações de saúde de uma determinada unidade de saúde municipal a uma OS.
Por esse serviço, o município paga à OS um valor estipulado em contrato e fiscaliza as metas de gestão contratadas. Entre as metas estabelecidas como variáveis no critério de remuneração, estão a média de permanência das internações e a taxa de ocupação dos leitos. O estímulo a internações mais curtas está no eixo central da política do Ministério da Saúde de indução de implantação de novos leitos de saúde mental em hospitais gerais: As diárias hospitalares se tornam decrescentes a partir do $8^{\circ}$ dia de internação e diminuem ainda mais após $16^{\circ} \mathrm{dia}$.

Botega e Dalgalarrondo agruparam as variáveis que interferem no tempo da internação em quatro grupos: variáveis clínicas e sociodemográficas dos pacientes; rede de serviços de saúde mental e sociais disponíveis; perfil da instituição que realiza a internação; e atitudes sociais em relação aos pacientes ${ }^{19}$. O entendimento do papel da internação como pontual, no atendimento à crise, também favorece as internações breves. Segundo um entrevistado: "O espírito da enfermaria é de enfermaria de crise, enfermaria de emergência. É tirar do quadro mais agudo e encaminhar para a rede do Município" (Gestor Local B).

A meta de internações mais curtas pode criar dilemas nas decisões técnicas e gerenciais das equipes e gestores. A transferência de pacientes das enfermarias de saúde mental do hospital geral para hospitais psiquiátricos pode ser um exemplo disso. Os gestores locais relataram situações nas quais os pacientes apresentaram melhora do seu quadro agudo, mas, por falta de estrutura psicossocial, permaneciam internados à espera de vagas em algum outro dispositivo da rede de assistência social ou de saúde. Em situações em que o paciente permanecia mais tempo internado, colocava-se em questão sua transferência para um hospital psiquiátrico da rede municipal.

A oferta de leitos de saúde mental em hospital geral e de leitos em hospitais psiquiátricos é um ponto nevrálgico da rede de saúde mental municipal, que trabalha com os dois tipos de internações. Esse sistema de gestão produz 
o que Botega e Dalgalarrondo chamaram de sistema duplo, que é a existência simultânea de unidades de internação psiquiátrica em hospitais gerais e hospitais psiquiátricos, gerando o encaminhamento de pacientes com perfis diversos para cada modalidade de serviço ${ }^{17}$.

No caso em estudo, a internação em hospital geral é chamada de primeira linha pelo gestor municipal:

[...] estamos trabalhando com a perspectiva de que eles são os leitos de primeira linha. É preferível internar ali do que internar nos hospitais psiquiátricos. A ideia é fazer a inversão do modelo. (Gestor Municipal).

Outra possibilidade para acolhimento de pacientes com necessidades psicossociais mais complexas seriam dispositivos com características residenciais. As residências terapêuticas brasileiras são destinadas para egressos de longas internações psiquiátricas. Entretanto, alguns pacientes internados nos leitos de saúde mental dos hospitais gerais, com prolongamento dos dias de internação, poderiam ter seu cuidado continuado em residências terapêuticas, o que evitaria a transferência para hospitais psiquiátricos onde correriam elevado risco de tornarem-se pacientes de longa permanência. Segundo Barbato e colaboradores ${ }^{\mathbf{1 8}}$, os modelos de dispositivos residenciais sofreram mudanças consideráveis nos últimos anos e, atualmente, as residências podem atender a uma variedade de funções, como: a provisão de serviços de reabilitação com limitação de tempo; a intervenção em crise como alternativa à internação em unidades para agudos; e locais de transição para vida independente após curto período de internação.

\section{O processo de cuidado}

Todos os informantes enfatizaram que a localização da enfermaria de saúde mental no âmbito de um hospital geral ampliava a possibilidade de cuidado integral do paciente. Os hospitais são equipados com recursos que facilitam a diferenciação diagnóstica entre quadros psiquiátricos e síndromes mentais com causalidade orgânica e permitem o cuidado ampliado das necessidades de saúde do paciente.

A integração da enfermaria de saúde mental com outros setores dos hospitais acontecia de duas formas: pela divisão de responsabilidade com os pacientes internados ou mediante divisão do profissional em mais de uma linha de cuidado do hospital. Quando os profissionais de saúde mental precisavam de um parecer de outra área, chamavam o especialista, e este auxiliava na orientação e condução do caso. Da mesma forma, quando outros profissionais precisavam de orientação da equipe de saúde mental, os profissionais iam até o setor e auxiliavam na direção do caso. Esse intercâmbio profissional acontecia em todas as unidades, contribuindo para integração da saúde mental com outras práticas do hospital.

A integração do setor de saúde mental com o restante do hospital geral é uma questão crucial. A literatura aponta que os pacientes com transtorno mental apresentam maior risco de desenvolver algumas comorbidades somáticas, como, por exemplo, diabetes, cardiopatias e doenças pulmonares, em decorrência do uso prolongado de medicações, como os antipsicóticos, e dos hábitos de vida não saudáveis, como uso de tabaco, falta de atividade física e alimentação inadequada ${ }^{19}$.

Nas enfermarias de saúde mental estudadas, o cuidado oferecido aos pacientes apresentava algumas variações, mas todas as três enfermarias se mostraram ambientes restritivos em relação ao seu funcionamento. Os usuários não podiam manter seus pertences, usar celulares e circular pelo hospital. As saídas desacompanhadas eram eventos esparsos. Os pertences ficavam guardados, podendo o paciente ter um conjunto de higiene e roupas íntimas. Em uma enfermaria, os pacientes podiam ficar com suas roupas, mas em duas enfermarias, eles usavam uniformes hospitalares. Alimentos trazidos de fora também eram proibidos em duas das três enfermarias. Em geral, as internações 
se restringiam à permanência dos usuários no espaço da enfermaria. As regras impostas para o funcionamento dos leitos de saúde mental parecem atender às necessidades de organização do hospital geral e de sua rotina. Os pacientes dos leitos de saúde mental são pacientes atípicos ao hospital geral. A maioria permanece a maior parte do tempo fora do leito. Andam pela enfermaria, conversam, fazem refeições fora das camas, necessitando de uma rotina de cuidados diferente da ofertada para as outras clínicas do hospital.

Tansella $^{20}$, em estudo que avaliou alternativas para a internação, aponta para a insatisfação de muitos usuários e profissionais com o atendimento hospitalar atual: eles demandam por um tratamento mais seguro e um ambiente mais amigável, com maior liberdade e menor distanciamento entre técnicos e pacientes. $\mathrm{O}$ autor pontua que o cuidado hospitalar na rede contemporânea de saúde mental é, provavelmente, o componente mais problemático do sistema. Uma revisão sistemática sobre a satisfação de usuários com serviços de internação mostrou que ela é maior quando os usuários são admitidos voluntariamente, em enfermarias abertas; e desenvolvem relações mais estreitas com a equipe. Por outro lado, experiências coercitivas durante a internação parecem desempenhar um papel central nas avaliações negativas ${ }^{21}$.

Os cuidados oferecidos pelas unidades se baseavam em atenção de enfermagem, psicofarmacologia e atendimentos individuais pelos técnicos. Os pacientes internados ficam sobre a lógica do cuidado da enfermagem, que supervisionam suas atividades de higiene, alimentação, medicação e sono. A presença de equipe multiprofissional não parece influir nessa prática. As poucas atividades desenvolvidas pela equipe multiprofissional não eram estruturadas e não pareciam influenciar a estrutura biomédica estabelecida, em que a farmacologia se confirmava como intervenção mais prevalente.

A Portaria GM $n^{\circ}$ 148/2012 determina que haja, pelo menos, dois profissionais de nível superior (não médicos e enfermeiros) na equipe da enfermaria ${ }^{7}$. Isso ocorria no Hospital Ronaldo Gazolla, enquanto no Hospital Evandro Freire havia apenas um psicólogo dedicado integralmente à enfermaria, e o Hospital Pedro II contava com um profissional de psicologia que se dividia entre a enfermaria e outros setores do hospital. O Hospital Ronaldo Gazolla possuía equipe maior (de médicos e demais profissionais) do que a dos outros hospitais e, assim como o Hospital Evandro Freire, não atendia emergências externas. A equipe de enfermagem estava completa em todas as três enfermarias, porém, a equipe de técnicos de enfermagem era inferior ao preconizado nas enfermarias de saúde mental dos hospitais Evandro Freire e Pedro II.

A heterogeneidade na composição das equipes é importante para garantir multiplicidade de olhares sobre a situação de crise vivida pelo sujeito e favorece a elaboração de um projeto terapêutico mais abrangente, alinhado as suas necessidades psicossociais. Nas entrevistas feitas com os gestores locais das enfermarias, o trabalho em equipe apareceu como forma de organização da assistência. As equipes dos três hospitais estão presentes de maneira diferenciada em decisões importantes, como confecção do projeto terapêutico do paciente, admissões e altas.

Esses achados estão de acordo com pesquisa desenvolvida por Gigantesco e colaboradores ${ }^{22}$ sobre as unidades hospitalares italianas. Segundo os autores, nas unidades psiquiátricas para tratamento de pacientes em situação de crise, existe um domínio da abordagem médica, com centralidade na terapia farmacológica. Foi constatado que uma menor oferta de equipe estava associada a uma política de portas trancadas. Um menor número de episódios de contenção física foi associado à oferta de psicoterapias individuais ou terapias comportamentais. A presença mais elevada de membros da equipe foi associada a uma maior disponibilidade de diferentes tipos de terapia (esse efeito foi mais evidente para os psicólogos), enquanto a presença e o número 
de regras foram, geralmente, associados com menos horas de trabalho da equipe.

As informações colhidas apontam para algumas deficiências no planejamento da estrutura física das unidades que impactam o processo de cuidado. As contenções mecânicas, quando necessárias, eram feitas nos quartos coletivos, exigindo maior atenção da equipe de enfermagem quanto à segurança do paciente. Um entrevistado, sobre a contenção mecânica, disse: "Fica na enfermaria, porque a gente não tem ambiente separado para isso. A enfermagem fica circulando e observando" (Gestor Local B). A contenção mecânica é reportada como um evento comum em enfermarias de saúde mental para pacientes em situação aguda, sendo geralmente utilizada quando existe a presença de agressividade que possa colocar em risco os pacientes e a equipe.

\section{A desinstitucionalização da atenção}

A falta de qualificação de profissionais para implantar os leitos de saúde mental nos hospitais gerais brasileiros apareceu nos depoimentos dos ex-coordenadores nacionais de saúde mental e do gestor municipal. A formação dos médicos e dos outros profissionais na graduação e na pós-graduação não vem acompanhando as mudanças no cenário assistencial e apresenta a psiquiatria e a saúde mental aos seus alunos como se fossem uma prática específica dos hospitais psiquiátricos.

Sobre isso, comenta um ex-coordenador nacional:

O Rio de Janeiro tem uma resistência histórica ao modelo de saúde mental no hospital geral. Se você ensina psiquiatria e saúde mental essencialmente dentro de hospital psiquiátrico, como você vai estimular a formação das pessoas para trabalharem no hospital geral? Eu atribuo a resistência a mudança do modelo a forte presença do hospital psiquiátrico no Rio de Janeiro e pela formação dos psiquiatras em hospital psiquiátrico. Isto se repete em São Paulo. (Ex-coordenador Nacional A).
[...] a dificuldade do Brasil fazer com que o hospital geral responda à questão da psiquiatria e da saúde mental é uma fragilidade da reforma psiquiátrica. Há um componente de desinformação, sem dúvida, há um componente da ineficiência dos programas de capacitação que foram feitos, não tiveram impacto. E há um componente importante que poderíamos chamar, com certo receio da palavra, de estigma. É o estigma contra o serviço de saúde mental. O hospital geral no Brasil pensa o serviço de saúde mental como um serviço de altíssima complexidade de funcionamento. E a experiência internacional de hospitais gerais é uma experiência que é totalmente ao contrário disso. (Ex-coordenador Nacional B).

Sobre o papel futuro do hospital psiquiátrico nos sistemas de atenção em saúde mental, os entrevistados corroboraram a ideia da superação do hospital psiquiátrico e sua substituição por uma rede de cuidados comunitários:

O nosso argumento ao longo do tempo, continua sendo, o mesmo: o cuidado deve ser provido em outro lugar. O transtorno mental não justifica internação em hospital psiquiátrico. (Excoordenador Nacional A).

Eu acho que os hospitais psiquiátricos não devem existir, eles são totalmente substituíveis por um sistema equilibrado com Caps III e hospital geral. (Ex-coordenador Nacional B).

O Brasil vinha adotando um modelo de transição com implantação progressiva de uma rede substitutiva ao mesmo tempo que promovia uma redução de leitos localizados em hospitais psiquiátricos. Esse processo foi refreado a partir do segundo semestre de 2016, quando o Ministério da Saúde alterou sua concepção em relação à matéria, passando a considerar os hospitais psiquiátricos como dispositivos efetivos da Raps conforme explicitado: 
A desinstitucionalização de moradores de hospitais psiquiátricos não é sinônimo de fechamento dessas instituições, pois há necessidade de leitos para internações de pacientes com quadro clínico grave. A busca da Política de Saúde Mental é qualificar o uso dessa opção de atendimento e garantir a melhoria na assistência hospitalar aos pacientes ${ }^{23}$.

Em sistemas nos quais a rede comunitária coexiste com os hospitais psiquiátricos, conformando um sistema dual, os pacientes com maior comprometimento psicossocial podem ser referidos preferencialmente a hospitais. A falta de consenso sobre o destino dos hospitais psiquiátricos é considerada uma das principais dificuldades do processo de desinstitucionalização. Além disso, em países que implementaram uma redução em larga escala no número de leitos em hospitais psiquiátricos sem a decisão do fechamento definitivo dos hospitais, a saída de todos os pacientes é apenas uma opção, e muitos podem permanecer hospitalizados ${ }^{24}$.

\section{Considerações finais}

A pesquisa pretendeu conhecer os aspectos envolvidos no modelo organizacional dos leitos de saúde mental em três hospitais gerais da cidade do Rio de Janeiro. A importância deste estudo se dá em função da política de saúde mental que está em curso no País desde a década de 1980, onde o leito de saúde mental em hospital geral deveria se destacar como componente hospitalar de atenção às crises mais complexas.

Cabe considerar, entretanto, que mesmo em países que substituíram os hospitais psiquiátricos por redes de atenção comunitárias e hospitais gerais, o cuidado hospitalar ainda representa uma questão central para formuladores de política, profissionais e usuários porque os padrões de cuidado and Contributor ID).
A cidade do Rio de Janeiro vem caminhando para a substituição do antigo modelo hospitalar pelo modelo de atenção comunitária. Entretanto, o cenário assistencial da cidade ainda é híbrido, com o hospital psiquiátrico ainda sendo apresentado como possibilidade de cuidado para pessoas em crise, condicionando perfis para as diferentes internações.

Apesar do grande quantitativo de hospitais gerais municipais, apenas quatro oferecem leitos de saúde mental. Historicamente, o Rio de Janeiro tinha uma significativa capacidade instalada de leitos em hospitais psiquiátricos localizada nos hospitais federais da antiga Divisão Nacional de Saúde Mental, herdados pela municipalidade. A oferta desses leitos parece ter funcionado como barreira para a implantação de leitos de saúde mental em hospitais gerais mais extensiva.

O município vem avançando em sua rede de Serviços Residenciais Terapêuticos, possibilitando que pessoas institucionalizadas possam voltar ao convívio comunitário de maneira assistida. Porém, o modelo de residências terapêuticas adotado pelo Ministério da Saúde e pelo município do Rio procura atender pessoas egressas de longo período de institucionalização, não servindo a pessoas sem suporte social, com menor permanência institucional. $\mathrm{O}$ estudo observou que as equipes das enfermarias não contavam com a retaguarda de serviços residenciais terapêuticos para casos com dificuldade de retorno à vida comunitária.

A baixa implantação de leitos de saúde mental em hospital geral parece estar relacionada com estigmas e preconceitos. A escassa informação sobre o assunto faz com que gestores resistam em receber pacientes com transtornos mentais em suas instituições, dificultando a reversão do modelo assistencial.

Como direção para pesquisas futuras, podem-se apontar a análise dos custos operacionais das enfermarias nos hospitais e 
sua comparação com os custos de outras estruturas assistenciais e estudos sobre as preferências dos usuários em relação aos dispositivos em que ocorre a atenção às crises, pois estes são os principais interessados nas consequências de decisões gerenciais e políticas.

\section{Referências}

1. Shen GC, Snowden LR. Institutionalization of deinstitutionalization: a cross-national analysis of mental health system reform. Int J Ment Health Syst. 2014; 8(1):47.

2. Brasil. Ministério da Saúde. Portaria GM nº 3088 . Institui a Rede de Atenção Psicossocial para pessoas com sofrimento ou transtorno mental e com necessidades decorrentes do uso de crack, álcool e outras drogas. Diário Oficial da União. 26 Dez 2011. [acesso em 2020 set 8]. Disponível em: http://bvsms.saude. gov.br/bvs/saudelegis/gm/2011/prt3088_23_12_2011_ rep.html.

3. Almeida JMC, Gonzalez FT. Atención Comunitaria a Personas com Trastornos Psicóticos. Washington, DC: OPS; 2005.

4. Ghio L, Favaretto G, Rocca G, et al. Residential Care in Italy: Critical Issues and Future Perspective. Int. J. Ment. Health. 2016; 45:32-41.

5. Botega NJ. Prática Psiquiátrica em hospital geral: interconsulta e emergência, 2. ed., Porto Alegre: Artmed; 2006.

\section{Colaboradores}

Echebarrena RC (0000-0002-7375-4124)* e Silva PRF (0000-0003-0811-4080)* contribuíram igualmente para a elaboração do manuscrito.
6. Desviat M, Moreno A. Las reformas tardias. In: Desviat M, Moreno A, editores. Aciones de salud mental en la comunidad. Madrid: AEN; 2012. p. 37-48

7. Brasil. Ministério da Saúde. Portaria MS/GM nº 148 . Define as normas de funcionamento e habilitação do Serviço Hospitalar de Referência para atenção a pessoas com sofrimento ou transtorno mental e com necessidades de saúde decorrentes do uso de álcool, crack e outras drogas. Diário Oficial da União, Brasília, DF. 31 Jan 2012. [acesso em 2020 set 8]. Disponível em: http://bvsms.saude.gov.br/bvs/saudelegis/ sas/2016/prt2650_29_12_2016.html.

8. Brasil. Ministério da Saúde. Mais de R\$ 2 milhões para ampliar rede de Saúde Mental. 2019. [acesso em 2020 fev 25]. Disponível em: https://www.saude.gov.br/noticias/agencia-saude/45927-mais-de-r-2-milhoes-para-ampliar-rede-de-saude-mental.

9. Fagundes Jr HM, Desviat M, Silva PRF. Reforma Psiquiátrica no Rio de Janeiro: situação atual e perspectivas futuras. Ciênc. Saúde Colet. 2016; 21(5):14491460. 
10. Creswell JW. Projeto de Pesquisa: Métodos qualitativo, quantitativo e misto. 2. ed. Porto Alegre: Artmed; 2007.

11. Brasil. Ministério da Saúde, Área técnica de saúde mental, álcool e outras drogas. Nota técnica 25/2012. Serviço hospitalar de referência para atenção em saúde mental.

12. Dias MK, Ferigato SH, Fernandes ADSA. Atenção à crise em saúde mental: centralização e descentralização das práticas. Ciênc. Saúde Colet. 2020; 25(2):595602 .

13. Barros REM, Tung TC, Mari JJ. Serviços de emergência psiquiátrica e suas relações com a rede de saúde mental Brasileira. Rev. Bras. Psiquiatria. 2010; 32(supl2):S71-S77.

14. Soranz D, Pinto LF, Penna GO. Eixos e a Reforma dos Cuidados em Atenção Primária em Saúde (RCAPS) na cidade do Rio de Janeiro, Brasil. Ciênc. Saúde Colet. 2016; 21(5):1327-1338.

15. Prata NISS, Groisman D, Martins DA, et al. Saúde mental e atenção básica: território, violência e o desafio das abordagens psicossociais. Trab. educ. saúde. 2017; 15(1):33-53.

16. Miliauskas CR, Faus DP, Junkes L, et al. Associação entre internações psiquiátricas, cobertura de CAPS e atenção básica em regiões metropolitanas do RJ e SP, Brasil. Ciênc. Saúde Colet. 2019; 24(5):1935-1944.

17. Botega N, Dalgalarrondo P. Saúde mental no Hospital Geral. Espaço para o psíquico. São Paulo: Hucitec; 1993.
18. Barbato A, Civenti G, D’Avanzo B. Community residential facilities in mental health services: A ten-year comparison in Lombardy. Health Policy. 2017; 121(6):623-628.

19. Prado MF, Sá MC, Miranda L. O paciente com transtorno mental grave no hospital geral: uma revisão bibliográfica. Saúde debate. 2015; 39(esp):320-337.

20. Tansella M. Alternatives to standard acute in-patient care for people with mental disorders: from systematic description to evaluative research. Br. J. Psychiatry. 2010; 197:(s53),s1-s3.

21. Woodward S, Berry K, Bucci S. A systematic review of factors associated with service user satisfaction with psychiatric inpatient services. Journal of Psychiatric Research J Psychiatr Res. 2017; 92:81-93.

22. Gigantesco A, Miglio R, Santone G, et al. Process of care in general hospital psychiatric units: national survey in Italy. J Psychiatry. 2007; 41(6):509-18.

23. Brasil. Ministério da Saúde. Governo incentiva menor permanência em hospitais psiquiátricos. 2018 [acesso em 2020 fev 24]. Disponível em: https://www.saude. gov.br/noticias/agencia-saude/44147-governo-incentiva-menor-permanencia-em-hospital-psiquiatrico.

24. Furlan PM, Zuffranieri M, Stanga F, et al. Four-Year Follow-Up of Long-Stay Patients Settled in the Community After Closure of Italy's Psychiatric Hospitals. Psychiat Serv. 2009; 60(9):1198-1202.

Recebido em 01/03/2020

Aprovado em 25/08/2020

Conflito de interesses: inexistente

Suporte financeiro: não houve 